\title{
Transgenic cereals by direct injection of DNA into plants
}

\section{Conrad Lichtenstein}

Currently there are two successful approaches that can be used to transform plants genetically, but each method has limitations that are delaying the application of the techniques to certain commercially important crops. On page 274 of this issue $^{1}, \mathrm{~A}$ de la Peña, H. Lörz and J. Schell report a significant step in applying a new method to cereals.

The first of the two current methods exploits a natural genetic engineer, Agrobacterium tumefaciens. This soil microbe contains a tumour-inducing (Ti) plasmid that transfers a DNA segment (the TDNA) from the plasmid to the nuclear genome of infected plants (or in vitro to plant tissue). The method is restricted to dicotyledenous plants (dicots); monocotyledenous plants (monocots) are usually not susceptible (see my recent News and Views article $^{2}$ and refs 3 and 4 for reviews). See last week's issue ${ }^{5}$, however, for an exception.

The second method involves direct transfer of DNA to plant protoplasts, prepared by enzymatic digestion of cell walls, for example by chemically stimulated uptake using polyethylene glycol ${ }^{6}$ or a highvoltage pulse (electroporation), generating transient 'holes' in the protoplast membrane? This method depends on a tissue culture system that allows regeneration of mature plants from protoplasts. Both methods use dominant selectable markers (for example, kanamycin resistance) to select for the transformed tissue which can then be screened for expression of co-transferred but unselected gene(s).

A major class of crops, the cereals, have so far evaded genetic manipulation. Because they are monocots they are not susceptible to agrobacterial infection and only in rice has it been possible to achieve plant regeneration from protoplasts. In this issue ${ }^{1}$, de la Peña and co-workers describe a novel approach to genetic transformation of cereals obviating the need for in vitro tissue culture and regeneration. The authors injected DNA encoding kanamycin resistance into the developing floral side-shoots (tillers) of rye plants (Secale cereale L.) and demonstrated that some progeny had acquired kanamycin resistance by incorporation of this DNA.

Within the floral tillers are archesporial cells; these give rise to pollen in the developing pollen sac by two meiotic cell divisions. Previous work by de la Peña $e t$ $a l .{ }^{8}$ had shown that these cells are permeable to colchicine at a stage 14 days before the first meiotic metaphase; colchicine disrupts meiosis and thus provides an assay for uptake.

This result suggested that such cells might also be able to take up larger molecules such as DNA. Thus, de la Peña $e t$ $a l .{ }^{1}$ injected a plasmid encoding kanamycin resistance, and under the control of a promoter known to drive transcription in plants, into the floral tillers of about 100 rye plants. After cross-pollination with other similarly injected plants the seeds were harvested and germinated on medium containing kanamycin. Of about 3,000 seeds so germinated, seven seedlings remained green, indicating resistance to kanamycin - sensitive seedlings bleach and die. Of these seven, further biochemical analyses showed that two had aminoglycoside phosphotransferase activity (encoded by the kanamycin resistance gene). DNA hybridization studies confirmed that this expression results from incorporation of the plasmid DNA. The plasmid DNA integrates in multiple copies, some of which show DNA rearrangements.

The selection method shows a background of kanamycin-resistant, yet untransformed, seedlings and the efficiency of transformation is low (about 0.07 per cent). A remaining question is whether unselected but co-injected DNA will integrate and express alongside the resistance gene at frequencies sufficient to allow detection by screening transformants.

Notwithstanding these provisos, one exciting prospect is that this simple transformation procedure can be applied to other cereals that currently lack transformation systems by DNA injection into similar pre-meiotic germline tissue.

\footnotetext{
1. de la Pena, A., Lorz, H. \& Schell, J.Nature 325, 274-276 (1987).

2. Lichtenstein, C. Nature 322, 682-683 (1986).

3. Lichtenstein, C. \& Draper, J. in DNA Cloning Vol. II (ed Glover, D. M.) Ch. 4 (IRL, Oxford, 1986)

4. Fraley, R. et al. CRC Crit. Rev. Plant Sci. 4, 1-46 (1986)

5. Grimsley, N., Hohn, T., Davies, J. W. \& Hohn, B. Nature 325, 177-179 (1987).

6. Paszkowski, J. et al. Meth. Enzym. 118, 668-684 (1986).

7. Fromm, M. et al. Meth. Enzym. (in the press).

8. de la Pena, A. et al. Chromosoma 83, 241-248 (1981).
}

Conrad Lichtenstein is in the Centre for Biotech nology, Imperial College of Science and Technology, Prince Consort Road, London SW7 $2 A Z, U K$.

\section{Antigen recognition Class discrimination in the world of immunology}

\section{Michael J. Bevan}

THE upheaval which occurred in the early 1970s with the demonstration that helper and cytotoxic $\mathrm{T}$ lymphocytes recognize foreign antigens only in association with self surface glycoproteins encoded by the major histocompatibility complex (MHC) continues to be felt. Although we have grown used to the idea that helper T cells recognize degraded (peptide) forms of soluble protein antigens in the context of class II MHC products, we now have to cope with the realization that class Irestricted cytotoxic $\mathrm{T}$ lymphocytes, which are usually specific for cellular antigens such as viral proteins, also recognize degraded forms of the antigen.

It makes biological sense to direct lytic $T$ cells towards the infected cell and to focus helper $T$ cells on soluble antigen in order to increase the production of antibodies that will neutralize toxins or viral particles. But if both subsets recognize peptide fragments of foreign antigen, how is this distinction made?

It now seems that it is the responsibility of the two classes of $\mathrm{MHC}$ molecules to distinguish between soluble, endocytosed antigens and cellular antigens. The way this is accomplished, as discussed recently by Germain in a News and Views article', may be by the non-random intracellular trafficking of vesicles that contain either class I or class II molecules. Vesicles that contain class II but not class I molecules intersect with the endocytic pathway and may present peptides derived from pinocytosed proteins. Class I-containing vesicles receive the peptide products derived from endogenously synthesized proteins. The latest information on the class I MHC-restricted recognition of integral cell membrane proteins is provided by two papers recently published in Nature ${ }^{2,3}$.

Previous work on the class I-restricted cytotoxic T-lymphocyte recognition of cells expressing influenza nucleoprotein established that fragments of the protein were recognized ${ }^{4.5}$. But this protein is made on free ribosomes. Thus, these experiments left open the question of whether integral membrane proteins, which are synthesized on membranebound ribosomes to be transported through the endoplasmic reticulum and Golgi complex and expressed in native form alongside class I molecules, would also be recognized as peptides by class Irestricted cytotoxic $\mathrm{T}$ lymphocytes. The 